\title{
A sum rule for charged elementary particles
}

\author{
Gerd Leuchs $^{1,2}$ and Luis L. Sánchez-Soto ${ }^{1,2,3, a}$ \\ 1 Max-Planck-Institut für die Physik des Lichts, Günther-Scharowsky-Straße 1, Bau 24, 91058 Erlangen, Germany \\ 2 Department für Physik, Universität Erlangen-Nürnberg, Staudtstraße 7, 91058 Erlangen, Germany \\ 3 Departamento de Óptica, Facultad de Física, Universidad Complutense, 28040 Madrid, Spain
}

Received 17 September 2012 / Received in final form 15 January 2013

Published online 21 March 2013

(C) The Author(s) 2013. This article is published with open access at Springerlink.com

\begin{abstract}
There may be a link between the quantum properties of the vacuum and the parameters describing the properties of light propagation, culminating in a sum over all types of elementary particles existing in Nature weighted only by their squared charges and independent of their masses. The estimate for that sum is of the order of 100 .
\end{abstract}

\section{Introduction}

The speed of light in vacuum and the impedance of the vacuum for electromagnetic radiation are experimentally determined parameters, the value of which has not been deduced so far. The same holds for the fine structure constant. Here, we use a simple model, borrowed from the description of dispersion in solid state physics, to attempt to establish a link between classical optics, i.e. Maxwell's equations, and the quantum properties of the vacuum.

Maxwell's displacement, $\mathbf{D}=\varepsilon_{0} \mathbf{E}+\mathbf{P}$, contains a quantity called the electric polarization of the vacuum. In the SI system, this quantity is $\varepsilon_{0} . \mathbf{P}$ describes the polarization of the medium, in case we are not dealing with just the vacuum. Normally, $\varepsilon_{0}$ is taken as a parameter given by Nature. In the past, its value has occasionally been adjusted with the availability of more precise measurement. Likewise, $1 / \mu_{0}$ is the magnetization of the vacuum ${ }^{1}$, $\mathbf{H}=\mathbf{B} / \mu_{0}-\mathbf{M}$. Here we expand on our earlier analysis [1] to underline its relevance for particle physics. A related

\footnotetext{
${ }^{a}$ e-mail: lsanchez@fis.ucm.es

1 Initially, quantities such as the speed of light and the impedance of the vacuum where experimentally determined parameters. Then, in the SI system in 1948 the value for $\mu_{0}$ was defined. Later, in 1983 the speed of light was given a defined value. As a result, $\varepsilon_{0}$ and the vacuum impedance also had defined values. These definitions were made jointly by the institutions in charge of standards world wide. The values were defined to be compatible with the earlier experimental values within the error bars. Currently, new SI definitions are being discussed by the same institutions with the goal to improve the standards e.g. of the kilogram. As a side effect, $\mu_{0}$ and $\varepsilon_{0}$ will be experimentally determined numbers again. For the purpose of this paper we, therefore, consider the above constants of classical electromagnetism to be experimental numbers, which may tell us something about Nature.
}

proposal linking the quantum vacuum to light propagation was obtained independently by Urban et al. [2].

In the early days of quantum mechanics, Weisskopf made the statement that the positron theory works well provided one ignores any electric and magnetic polarizability of the vacuum it may imply [3]. Looking back, we would reinterpret this statement as meaning that the polarizability of the virtual electron-positron pairs in the vacuum must, of course, be already contained in Maxwell's equations - otherwise they would not work so well - and it would be wrong to account for the same effect a second time. However, this implies the properties of the quantum vacuum govern the propagation of light and thus govern all of classical optics. Heitler [4] likewise mentions that $\varepsilon_{0}$ may be thought of as the polarizability of the vacuum associated with the electric dipoles induced in the virtual electron-positron pairs by an external electric field. We now take this literally and relate the parameters appearing in Maxwell's equations, $\varepsilon_{0}$ and $1 / \mu_{0}$, to the quantum properties of the vacuum. Incidentally, the term Maxwell added to form the Lorentz invariant set of equations, he interpreted as the displacement current of the vacuum. In our approach, this interpretation comes to life, resulting in a Lorentz invariant contribution of the quantum vacuum to the propagation of light.

\section{The model}

The speed of light plays a multiplicity of roles in considerations describing different physical quantities: (1) $c_{\mathrm{rel}}$, the relativistic relation between the mass of a particle and its rest energy and the limiting speed in the Lorentz transformation; and (2) $c_{\text {light }}$, the phase velocity of electromagnetic radiation in vacuum. For the argument below, we first keep $c_{\text {rel }}$ and $c_{\text {light }}$ as separate and not necessarily 
identical quantities. This obviously means that, for the moment, we relax the requirement for Lorentz invariance of Maxwell's equations. We derive the speed of light and the impedance of the vacuum on the basis of the properties of the quantum vacuum treating it as a dielectric and diamagnetic medium and then compare these values to the experimentally observed ones, thus restoring Lorentz invariance. We will see that the impedance depends on the sum over the squared charges of the different types of elementary particles, while the speed of light is independent of this sum. The latter underlines the general nature of the speed of light. According to present day knowledge, the sum reads

$$
\sum_{j}^{e . p .} \frac{q_{j}^{2}}{e^{2}}=1+1+1+3\left(3 \frac{1}{9}+3 \frac{4}{9}\right)+1+\ldots ?
$$

The sum has to account for all types of elementary particle-antiparticle pairs, known and unknown. The known ones sum up to 9, accounting for electron, muon, tauon, six different quarks each coming in three colour charges, as well as the charged $W$-boson.

The model of the vacuum with dielectric and diamagnetic properties described below is clearly oversimplified and a more rigorous model is needed. But it will help us getting a first insight into the relation between the quantum vacuum and optics.

\subsection{The polarization of the vacuum}

The vacuum is assumed to consist of virtual particleantiparticle pairs treated as extremely short-lived polarizable objects. The polarization is a dipole moment density; therefore, one has to calculate the dipole moment induced by an external electric field and divide by the volume occupied by the pair. We have two ways of calculating the induced dipole moment.

The first possibility is to suppose there is a spring holding a virtual pair together. The spring constant should be related to the energy needed to excite the virtual pair to a real pair $\hbar \omega_{0}=2 m c_{\text {rel }}^{2}$. Since optical frequencies are a million times smaller than the frequency associated to the electron-positron energy gap, we are essentially dealing with the static limit of the driven harmonic oscillator. Note that two equal harmonically bound masses $m$ correspond to a harmonic oscillator with only one mass given by the reduced mass $m_{\text {red }}=m / 2$. The corresponding induced electric dipole moment is

$$
d=e x=\frac{e^{2}}{m_{\mathrm{red}} \omega_{0}^{2}} \zeta E=\frac{2 e^{2}}{m \omega_{0}^{2}} \zeta E=\frac{e^{2} \hbar^{2} \zeta}{2 m^{3} c_{\mathrm{rel}}^{4}} E .
$$

The factor $\zeta$ accounts for transient creation of the electric dipole; i.e. for averaging over the initial transient dynamics leading to an electric dipole moment smaller than the static limit.

The volume occupied by a single virtual pair can be estimated using the uncertainty relation and should thus be of the order of the cube of the Compton wavelength of the electron:

$$
V=\eta\left(\frac{\hbar}{m c_{\mathrm{rel}}}\right)^{3} \text {. }
$$

We allow for some flexibility by introducing an additional factor $\eta$, which we expect to be of order unity. The polarization of the electron-positron vacuum is thus

$$
P_{0}=\frac{d}{V}=\frac{e^{2} \zeta}{2 c_{\mathrm{rel}} \hbar \eta} E .
$$

Since the mass drops out, different types of elementary particles having the same electric charge contribute equally to the vacuum polarizability irrespective of their mass. Hence, to obtain the full vacuum response we have to sum over all types of elementary articles, known and unknown:

$$
P_{0}=\frac{\zeta}{2 c_{\mathrm{rel}} \hbar \eta}\left(\sum_{j}^{e . p .} q_{j}^{2}\right) E \equiv \varepsilon_{0} E .
$$

As mentioned before, the static limit may be too large an estimate for the induced dipole moment, because the charges have to be accelerated to this value. The factor $\zeta$ was introduced to account for this dynamical polarization process. Starting from zero and averaging over the transiently appearing dipole moment for a time given by the uncertainty relation for the particle-antiparticle pairs, one obtains $\zeta=0.15$. Furthermore, we take the scale factor $\eta$ the same for all particles.

Within this model one may wonder about a possible frequency dependence of the vacuum polarization as a result of the resonances at the rest mass energies. However, in a real excitation the conservation of momentum should be fulfilled, prohibiting the excitation of a virtual pair to a real pair in free space with a plane wave. Far away from resonance, the process is allowed because of the quantum uncertainty of the momentum. Far above resonance, we would expect the induced dipole moment to decrease as $1 / \omega_{0, j}^{2}$. In contradistinction, a converging dipole wave may excite real pairs in the vacuum [5].

The second alternative of calculating the induced dipole moment is to take the particle and the antiparticle to be free. Accordingly, the two particles would be accelerated in the external electric field in opposite directions, but only for the ultra short time during which we can consider the virtual pairs to exist, which is given by the relation $\Delta \mathscr{E} \Delta t \geq \hbar$. The time interval is thus $\Delta t \simeq \hbar / 2 m_{j} c_{\mathrm{rel}}^{2}$. This leads to the same expression as in equation (5). Integrating over time in this free-particle model yields $\zeta=0.17$, slightly larger than the value obtained in the harmonic oscillator model above.

\subsection{The magnetization of the vacuum}

Next, we need to develop the same procedure for the magnetic response. The induced magnetic dipole moment 
$d_{\text {magn }}$ is given by the current induced in a loop multiplied by the loop area:

$$
d_{\text {magn }}=2 i A=2\left(q_{j} \nu\right)\left(\pi \varrho^{2}\right) .
$$

The factor 2 comes about because the oppositely charged particle and antiparticle both contribute equal amounts. The frequency at which the charge goes around the loop is the cyclotron frequency:

$$
d_{\text {magn }}=\frac{q_{j}^{2}}{m_{j}} \varrho_{j}^{2} B .
$$

The average radius of the current loop $\varrho_{j}$ is of the order of the Compton wavelength, with a scale factor $\xi$ also taken to be independent of the particle type:

$$
\varrho_{j}^{2}=\xi\left(\frac{\hbar}{m_{j} c_{\mathrm{rel}}}\right)^{2} .
$$

Dividing by the volume of the virtual pair (Eq. (3)) we obtain the vacuum magnetization

$$
M_{0}=\frac{\xi c_{\mathrm{rel}}}{\eta \hbar}\left(\sum_{j}^{e . p .} q_{j}^{2}\right) B \equiv \frac{1}{\mu_{0}} B .
$$

Again the mass drops out and we sum over all types of elementary particles. We assume the vacuum to be diamagnetic: the particle-antiparticle pairs will be in singlet states and there will be no contribution of the total spin of each pair to the magnetization of the vacuum ${ }^{2}$.

\subsection{The speed of light and the impedance of the vacuum}

Now, the stage is set to relate the speed of light $c_{\text {light }}$ and the impedance of the vacuum $Z_{0}$ to the properties of the quantum vacuum. In Maxwell's theory $c_{\text {light }}=1 / \sqrt{\varepsilon_{0} \mu_{0}}$ and we can insert the model values for $\varepsilon_{0}$ and $\mu_{0}$ using equations (5) and (9):

$$
c_{\text {light }}=c_{\text {rel }} \sqrt{\frac{2 \xi}{\zeta}}
$$

Likewise, we find

$$
Z_{0}=\frac{\sqrt{2} \eta \hbar}{\sqrt{\xi \zeta}}\left(\sum_{j}^{e . p .} q_{j}^{2}\right)^{-1}=5811[\Omega] \frac{\eta}{\sqrt{\xi \zeta}}\left(\sum_{j}^{e \cdot p \cdot} \frac{q_{j}^{2}}{e^{2}}\right)^{-1}
$$

Several things deserve mentioning. First of all, if we set the scale factors to one and the sum over the normalized

\footnotetext{
${ }^{2}$ If, however, we associate a magnetic moment with each particle separately then the antiparallel spins will lead to parallel yet isotropic magnetic moments. In a way, we are making assumptions about the angular momentum coupling scheme when neglecting any paramagnetic contribution.
}

charges to nine, we get a speed of light and an impedance which are both off by only a factor of two. We consider this to be remarkably close and supporting the general applicability of the model. Secondly, the speed of light comes out to be independent of how many types of elementary particles contribute to the polarization and magnetization of the vacuum. This seems to underline the global characteristics of $c_{\text {light }}{ }^{3}$. We next use the experimental observation that $c_{\text {light }}=c_{\text {rel }}$; this yields

$$
\frac{\zeta}{\xi}=2 .
$$

Incidentally, this result can also be derived through requiring that the polarization and magnetization be the same in all frames. Thus, the expression for the impedance simplifies to

$$
Z_{0}=\frac{2 \eta \hbar}{\zeta}\left(\sum_{j}^{e \cdot p \cdot} q_{j}^{2}\right)^{-1}=8218[\Omega] \frac{\eta}{\zeta}\left(\sum_{j}^{e \cdot p \cdot} \frac{q_{j}^{2}}{e^{2}}\right)^{-1}
$$

\subsection{The fine structure constant}

The fine structure constant $\alpha$ relates to the strength of the coupling between the electromagnetic field and matter and is given by

$$
\alpha=\frac{e^{2}}{4 \pi \varepsilon_{0} \hbar c} .
$$

This is the zero-energy value. There is experimental evidence for an increase of $\alpha$ toward higher energies [6], referred to as the running fine structure constant. This is usually ascribed to the renormalization of the electric charge.

In quantum electrodynamics, one could modify $\varepsilon_{0}$ instead of renormalizing the electric charge. Our model suggests doing exactly this by relating $\varepsilon_{0}$ to the sum over the different types of elementary particles

$$
\varepsilon_{0}=\frac{\zeta}{2 c_{\mathrm{rel}} \hbar \eta}\left(\sum_{j}^{e . p .} q_{j}^{2}\right),
$$

which results in the following expression for the zeroenergy value of the fine structure constant:

$$
\alpha_{0}=\frac{\eta}{2 \pi \zeta}\left(\sum_{j}^{e \cdot p \cdot} \frac{q_{j}^{2}}{e^{2}}\right)^{-1} .
$$

The model prediction for $\alpha_{\mathscr{E}}$ at higher energies is obtained by omitting those particle-antiparticle pairs having a rest mass energy lower than $\mathscr{E}$. This allows for an alternative route to estimate the sum in equation (1).

\footnotetext{
${ }^{3}$ It may be worth noting that there seems to be an interesting analogy with a completely different quantity, namely the speed of sound in a gas, being largely independent of the density.
} 


\subsection{Dispersion}

One consequence of this model is that as soon as the light frequency increases beyond the gap frequency for one particular particle-antiparticle pair, the contribution of this pair to the sum will decrease ${ }^{4}$, both for $\varepsilon_{0}$ and $1 / \mu_{0}$. Within this model the sum cancels out when calculating the speed of light, predicting a frequency independent speed of light equal to $c_{\text {rel }}$. The vacuum impedance however will be affected starting at gamma ray frequencies above $1 \mathrm{MeV}$. It would certainly be interesting to investigate this prediction ${ }^{5}$.

\section{Discussion}

There are two independent comparisons between the model and experimental values both leading to a prediction for the sum over all charged particles, known and unknown ones.

\subsection{The number of charged elementary particles as derived from the impedance of the vacuum}

We can now set the model result in equation (13) for the vacuum impedance equal to the empirical value $Z_{0}=376.7 \Omega$. The remarkable result is that this provides information about future additional types of charged particle-antiparticle pairs:

$$
\sum_{j}^{e \cdot p \cdot} \frac{q_{j}^{2}}{e^{2}}=\frac{2 \eta \hbar}{e^{2} Z_{0} \zeta}=21.82 \frac{\eta}{\zeta}
$$

There are still two unknowns: the sum and $\eta$. Without any further information we use the initial assumption in Section 2.1 that $\eta \simeq 1 \pm \delta \eta$ :

$$
\sum_{j}^{e \cdot p \cdot} \frac{q_{j}^{2}}{e^{2}} \simeq 109(1 \pm \delta \eta) .
$$

Here, $\delta \eta$ accounts for the uncertainty in the value of $\eta$.

\footnotetext{
${ }^{4}$ When the frequency of the electromagnetic wave $\omega$ increases beyond the rest mass energy of one type of particleantiparticle pair, then the contribution of this type of particles to the sum in equation (1) drops to zero at a rate proportional to the inverse of the frequency squared for the electric polarizability and at a rate proportional to the inverse of the frequency for the magnetic polarizability. The electric dipole moment is induced with some delay owing to the inertia of the particle mass, while the magnetic dipole moment is induced instantaneously for a point charge. Thus, one would not expect any frequency dependence of the magnetic polarizability of point charges. However, the position uncertainty of the order of the Compton wavelength leads to a reduced current at frequencies higher than the resonance and thus to the inverse frequency dependence.

${ }^{5}$ If the statement about the different frequency dependences in the electric and in the magnetic case in footnote 4 is correct, there might be deviations to the speed of light in the vicinity of the rest mass energies.
}

\subsection{The number of charged elementary particles as derived from the energy dependence of the fine structure constant}

A second independent estimate for the sum over all particles involves the fine structure constant and its established variation with energy. The fine structure constant is $1 / 137.04$ at low energies and reduces to $1 /(128.5 \pm 2.5)$ at $58 \mathrm{GeV}$. At this energy we are beyond the rest mass energies of most of the well-known particle-antiparticle pairs (except for the top quarks and the $W$-bosons). So, omitting the particle types with $m_{j} c^{2}<58 \mathrm{GeV}$ the sum would reduce by $20 / 3=6.67$, increasing $\alpha$ correspondingly. Based on our model, and with the experimental values for $\alpha_{0}$ and $\alpha_{58} \mathrm{GeV}$, we find a second independent way to determine the sum:

$$
\begin{gathered}
\alpha_{0}^{-1}=137.04=\text { const. } \sum_{j}^{e . p .} \frac{q_{j}^{2}}{e^{2}} \\
\alpha_{58 \mathrm{GeV}}^{-1}=128.5 \pm 2.5=\text { const. } \sum_{j}^{\text {e.p.>58 GeV }} \frac{q_{j}^{2}}{e^{2}} .
\end{gathered}
$$

In the calculation, we can take into account that the contribution of one particle type does not fall off abruptly but proportional to $1 / \omega$, so each term in the sum is replaced with

$$
\frac{q_{j}^{2}}{e^{2}} \mapsto \frac{q_{j}^{2}}{e^{2}} \times \begin{cases}1 & \hbar \omega \leq m_{j} c^{2} \\ \left(\frac{m_{j} c^{2}}{\hbar \omega}\right)^{2} & \hbar \omega>m_{j} c^{2} .\end{cases}
$$

This results in a reduction of the sum for $58 \mathrm{GeV}$ by 6.5 instead of 6.67. Consequently,

$$
\frac{\alpha_{0}^{-1}}{\alpha_{58 \mathrm{GeV}}^{-1}}=\frac{\sum_{\text {all }}}{\sum_{>58 \mathrm{GeV}}}=\frac{\sum_{\text {all }}}{\sum_{\text {all }}-6.5}=\frac{137.04}{128.05 \pm 2.5} \text {, }
$$

so finally

$$
\sum_{\text {all }}=104\left\{\begin{array}{l}
+43 \\
-24
\end{array}\right.
$$

\subsection{Comparison}

The two different results obtained so far agree quite well. It seems that the approach in Section 3.2 is less ambiguous than the one in Section 3.1. One could use equation (21) to reduce this ambiguity in Section 3.1. Using $\zeta=0.2$ and imposing the result in (21) would lead to $\eta=1.05 \pm$ 0.31 . This number is very close to the one assumed in Section 3.1. Consequently, when using $\zeta=0.2, \xi=0.1$ and $\eta=1.05$, the two approaches are both compatible, the sum being of the order of 100 . This would predict many still undiscovered charged elementary particles with rest mass energies above $58 \mathrm{GeV}$. 


\section{Conclusions}

Our model is a most simple one and the quantitative results, namely the sum over the different types of elementary particles, have thus to be taken with caution. One feature of this model is that it relates the number of charged elementary particles to low-energy properties of the electromagnetic field, such as the vacuum impedance and the fine structure constant. The zero energy value of the fine structure constant, or equivalently the vacuum permittivity, has so far been a purely experimental number. As to the speed of light, the value predicted by the model is determined by the relative properties of the electric and magnetic interaction of light with the quantum vacuum and is independent of the number of elementary particles, a remarkable property underlining the general character of the speed of light.

Thus, the purpose of the simple model is to point at the intimate relationship between the properties of the quantum vacuum and the constants in Maxwell's equations. Indeed, from this picture, the vacuum can be understood as an effective medium [7]. Furthermore, we have devised two independent ways of checking the model predictions against the experimental values. We hope that this result will stimulate more rigorous quantum field theoretical calculations.

It is a pleasure acknowledging helpful discussions with Fabio Biancalana, Joseph H. Eberly, Holger Gies, Nicolai B. Narozhny, Klaus Rith, Wolfgang P. Schleich, Michael Thies,
Marcel Urban, François Couchot and Xavier Sarazin, as well as with the participants of the 500th Heraeus Seminar on "Highlights of Quantum Optics" at the Physik-Zentrum in Bad Honnef, May 6-11, 2012.

\section{References}

1. G. Leuchs, A.S. Villar, L.L. Sánchez-Soto, Appl. Phys. B 100, $9(2010)$

2. M. Urban, F. Couchot, X. Sarazin, arXiv:1106.3996 [physics.gen-ph], arXiv:1111.1847 [physics.gen-ph]

3. V. Weisskopf, in Selected papers on Quantum Electrodynamics, edited by J. Schwinger (Dover, New York, 1958), pp. $92-128$

4. W. Heitler, in The Quantum Theory of Radiation, 3rd edn. (Oxford University Press, Oxford, 1954), p. 113

5. N.B. Narozhny, S.S. Bulanov, V.D. Mur, V.S. Popov, Phys. Lett. A 330, 1 (2004)

6. I. Levine et al., Phys. Rev. Lett. 78, 424 (1997)

7. R.H. Dicke, Rev. Mod. Phys. 29, 363 (1957)

Open Access This is an open access article distributed under the terms of the Creative Commons Attribution License (http://creativecommons.org/licenses/by/3.0), which permits unrestricted use, distribution, and reproduction in any medium, provided the original work is properly cited. 